\title{
The Tobacco Status Project (TSP): Study protocol for a randomized controlled trial of a Facebook smoking cessation intervention for young adults
}

\author{
Danielle E. Ramo ${ }^{1,2^{*}} \mathbb{D}$, Johannes Thrul', Kevin L. Delucchi', Pamela M. Ling ${ }^{2,3}$, Sharon M. Hall ${ }^{1}$ and Judith J. Prochaska ${ }^{4}$
}

\begin{abstract}
Background: Tobacco use remains the leading cause of premature morbidity and mortality in the United States. Young adults are less successful at quitting, use cessation treatment less often than smokers of other ages, and can be a challenge to retain in treatment. Social media, integrated into the lives of many young adults, represents a promising strategy to deliver evidence-based smoking cessation treatment to a large, diverse audience. The goal of this trial is to test the efficacy of a stage-based smoking cessation intervention on Facebook for young adults age 18 to 25 on smoking abstinence, reduction in cigarettes smoked, and thoughts about smoking abstinence.

Methods/Design: This is a randomized controlled trial. Young adult smokers throughout the United States are recruited online and randomized to either the 3 month Tobacco Status Project intervention on Facebook or a referral to a smoking cessation website. The intervention consists of assignment to a secret Facebook group tailored to readiness to quit smoking (precontemplation, contemplation, preparation), daily Facebook contacts tailored to readiness to quit smoking, weekly live counseling sessions, and for those in preparation, weekly Cognitive Behavioral Therapy counseling sessions on Facebook. Primary outcome measure is biochemically-verified 7-day point prevalence abstinence from smoking at posttreatment (3 months), 6 , and 12 months. Secondary outcome measures are reduction of $50 \%$ or more in cigarettes smoked, $24 \mathrm{~h}$ quit attempts, and commitment to abstinence at each time point. A secondary aim is to test, within the TSP condition, the effect of a monetary incentive at increasing engagement in the intervention.
\end{abstract}

Discussion: This randomized controlled trial is testing a novel Facebook intervention for treating young adults' tobacco use. If efficacious, the social media intervention could be disseminated widely and expanded to address additional health risks.

Trial registration: ClinicalTrials.gov: NCT02207036, May 13, 2014.

\section{Background}

Cigarette smoking is the single most important preventable cause of morbidity, mortality, and excess health cost in the United States (US), accounting for 480,000 premature deaths each year $[1,2]$. Although the prevalence of cigarette smoking has declined among adults in the US since 1983, smoking among young adults aged $18-25$ years

\footnotetext{
* Correspondence: Danielle.Ramo@ucsf.edu

'Department of Psychiatry, University of California, 401 Parnassus Avenue,

Box TRC 0984, San Francisco, CA 94143, USA

${ }^{2}$ Center for Tobacco Control Research and Education, University of California,

530 Parnassus Avenue, San Francisco, CA 94143, USA

Full list of author information is available at the end of the article
}

has remained stable, with past month cigarette use rates as high as $31 \%$ in 2013 [3]. Almost all smokers (98\%) report starting before the age of 26 years [4], and more than 2000 US youth and young adults become daily cigarette smokers each day $[4,5]$.

Despite comparable smoking prevalence and proportion motivated to quit, relative to other adult age groups, young adults are less likely to use interventions for smoking cessation [6]. Further, studies of tobacco use and other health behaviors have reported great challenges in recruiting young adults $[7,8]$. Online smoking cessation strategies (e.g., websites designed to help people quit smoking) reach large numbers of smokers [9] but 
tend not to have a personalized approach to smoking cessation or to have follow-up treatment contacts [10]. Young adults are less likely to take advantage of these cessation resources, and studies of web-based smoking cessation programs have been associated with large drop-offs in engagement throughout the course of participation [11-14]. Websites directly targeted to young adults have typically focused on college students $[15,16]$ who are less likely to smoke than non-college bound young adults $[17,18]$. There is a need to develop innovative smoking cessation interventions to engage a wide audience of young adult smokers.

Social media represents a promising strategy to deliver evidence-based smoking cessation interventions to young adults. Social media tools are widely popular among young adults ( $89 \%$ of 18 to 29 year old Americans use the internet) [19] and can be harnessed to widely disseminate information about a broad range of behavioral and emotional changes including smoking cessation [20-23]. Facebook remains the most widely used social media tool by young adults in the United States. With $87 \%$ of US online young adults having a Facebook account and $70 \%$ of those accessing it daily [19], there is promise to use this platform to deliver public health intervention programs to young people. Previous evaluations using Facebook to change health risk behavior have shown feasibility as measured by participant's engagement and satisfaction [24-32]. However, trials examining social media interventions have shown limited or no effects on health behavior change (e.g., physical activity) [33]. The BIO smoking cessation campaign for young adults in Canada, incorporating a website, Smartphone app and Facebook features, resulted in greater 7 - and 30-day reported quit rates than referral to a Smokers' Helpline at 3 month follow-up [34]. Research is needed to determine whether Facebook alone can be used as an intervention tool, whether abstinence can be biochemically verified, and whether abstinence rates can be maintained over 1 year.

Our group conducted a mixed-methods study to determine how young adult smokers would like to use Facebook to help change smoking behavior. About a third (31\%) of survey respondents reported they would want to quit smoking using Facebook, and interest was greater among those more motivated to quit, who had made a quit attempt in the past year, and had previously used the Internet for assistance with a quit attempt (all $p<0.01$ ). In qualitative interviews, social support and convenience were identified as strengths of a Facebook intervention, while privacy was the main concern [35].

Based on this formative work, we developed the Tobacco Status Project (TSP), a Facebook intervention for young adult smokers combining Facebook contacts tailored to participants' readiness to quit smoking with a 12-week cessation program consisting of 90 days of Facebook postings, weekly counseling sessions, and for those ready to quit, 7 state-of-the-art group cognitive-behavioral sessions. A feasibility trial enrolled 79 young adults who formed 7 Facebook groups [36]. Follow-up rates were 84 and $72 \%$ at 6 and 12 months, respectively, and reported 7day abstinence was $21 \%$ at 6 months (9\% biochemicallyverified) and $18 \%$ at 12 months (9\% verified) (Ramo DE, Chavez K, Delucchi KL, Prochaska JJ: Feasibility and quit rates of the "Tobacco Status Project", submitted). From baseline to 12-months, there was a significant increase in the proportion prepared to quit (13 to $46 \%, \mathrm{p}<.001$ ), $35 \%$ reduced their cigarette consumption by $50 \%$ or greater, and $67 \%$ reported a 24-h quit attempt. Engagement in the intervention was high, with $92 \%$ participation in the full 3 month intervention and $61 \%$ commented on at least one post, with more commenting among those randomized to receive a personal monetary incentive [37]. Participants reported reading most of the Facebook posts (mean usability rating $=3.3 / 4$ ) and interactions from counseling sessions $(3 / 4)$, thinking about what they read (3/4) and would recommend the program to others $(3.3 / 4)$. Herein, we describe a randomized controlled trial testing the efficacy of TSP against a referral control condition with 480 young adult smokers who use Facebook.

\section{Aims and hypotheses}

Main Aim: To evaluate the efficacy of the Facebook-based intervention for young adults in a randomized controlled trial

Hypotheses are:

1. Participants receiving the intervention will have higher 7-day point prevalence abstinence at 3-, 6-, and 12-months follow-up compared to those in the control condition.

2. Participants in the intervention condition will demonstrate greater reduction in cigarettes smoked and increased commitment to abstinence at 3-, 6-, and 12-months follow-up than those in the control condition.

3. Participants in the intervention condition will be more likely to make a quit attempt during the 12-month study period compared to those in the control condition.

Secondary Aim: To evaluate effectiveness of a monetary incentive for engagement in the Tobacco Status Project intervention

Analyses will examine:

1. Engagement by incentive group (daily, weekly, monthly, no incentive); 
2. Abstinence rates by incentive group; and

3. Effect of engagement on abstinence

\section{Methods/Design}

\section{Overview of design}

This study is a randomized controlled trial (RCT) with 480 young adults age 18 to 25 recruited through Facebook (Fig. 1). Participants are randomized to one of two conditions: (1) the Tobacco Status Project motivationallytailored smoking cessation intervention delivered through Facebook (TSP); or (2) a control condition (referral to the Smokefree.gov website). Assessments are conducted online at baseline, 3-, 6-, and 12-months follow-up. Primary outcome is biochemically-verified 7-day point prevalence abstinence from tobacco, and secondary outcomes are reduction in cigarettes smoked per week, $24 \mathrm{~h}$ quit attempts, and commitment to abstinence in each time period. Within the TSP group, a monetary engagement will be tested as a moderator of engagement and abstinence.

\section{Inclusion criteria}

Participants are required to meet the following criteria in order to be eligible for enrollment in the trial:

1) English literate (at an $8^{\text {th }}$ grade reading level);

2) age 18 to 25 years (consistent with the definition of young adulthood used by the National Survey of Drug Use and Health, and age range with the highest smoking prevalence nationally [38]);

3) access to a digital camera that can take and send a picture (e.g., camera phone);

4) go on Facebook "most" $(\geq 4)$ days per week;

5) $\geq 100$ cigarettes smoked in their lives; and currently smoking at least 1 cigarette per day on 3 or more days of the week. Smoking criteria are based on those used in the National Health Interview Survey and are liberal based on smoking patterns most common in young adults and to include ethnic/ racial minority populations, who tend to smoke fewer cigarettes per day than Caucasians [1].

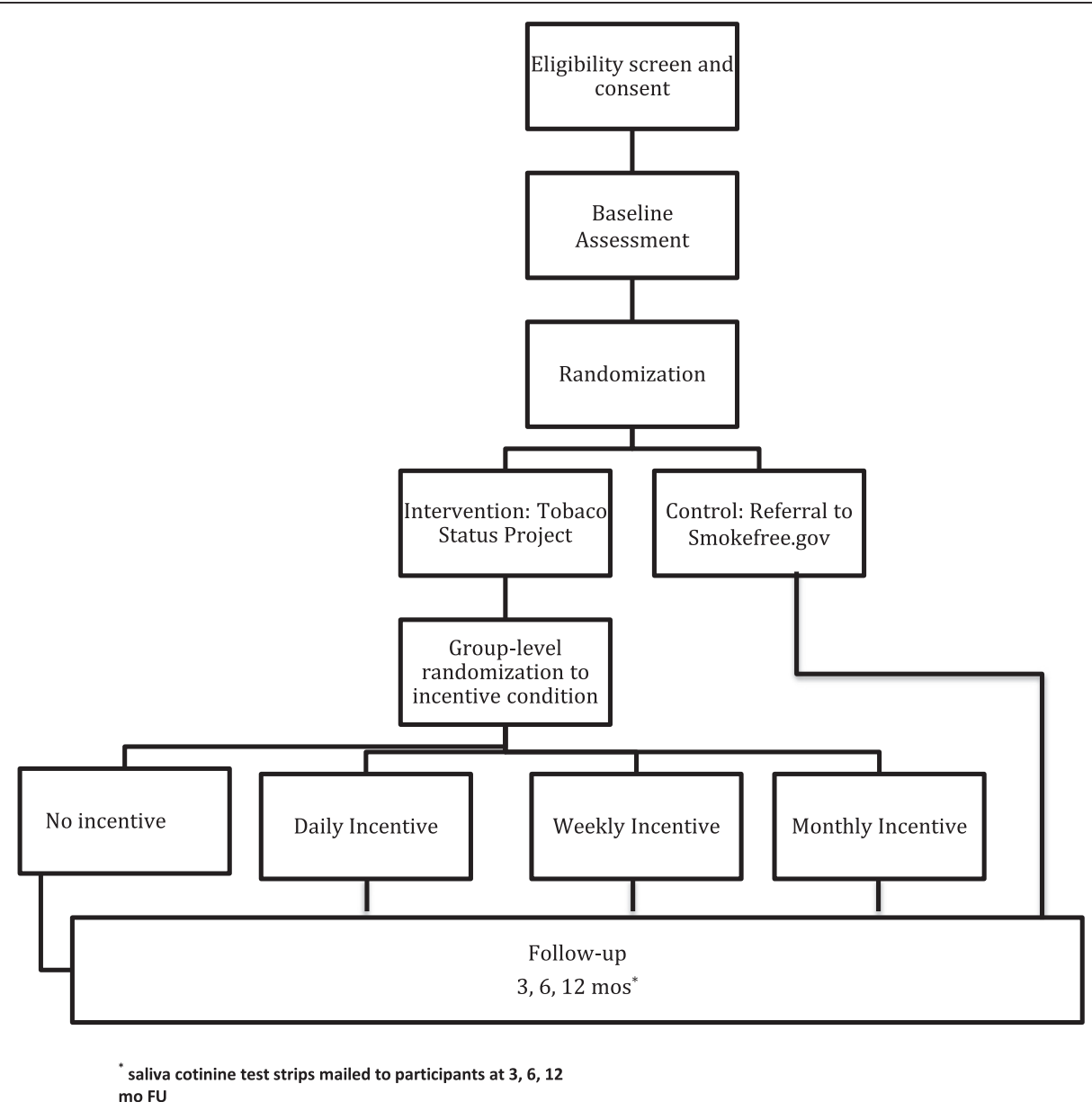

Fig. 1 Flow of participants through trial 


\section{Exclusion criteria}

1) previous participation in the TSP feasibility trial.

\section{Recruitment}

Figure 1 shows the flow of participants through the trial. Participants are being recruited over 16 months primarily from Facebook based on a media campaign that has been successfully employed by the team in previous online survey and interventions studies [36, 39, 40]. Facebook advertisements are targeted based on: 1) age (18 to 25$)$; 2) location; 3) gender; 4) race/ethnicity; and 4) keywords related to tobacco use. Ads include an image and short text consistent with Facebook's advertising guidelines. Most ads mention the study incentive. Facebook's Ads Manager program tracks progress of all advertisements in a campaign based on budget, numbers of clicks on an ad, and likelihood of enrollment in a study. Ads include a link to the study's independent Qualtrics website with a short description of the study and eligibility questions. If respondents are eligible, then they will be taken to the study's informed consent page.

\section{Study procedure}

Informed consent is obtained from all study participants in accordance with the Helsinki Declaration and approved by the UCSF Committee in Human Research (\#11-06294). During the informed consent process, participants' understanding of the information provided is assessed through a series of multiple choice questions with required responses before being able to proceed with the study. Questions are based on those used in studies with potentially vulnerable populations [41, 42]. Any wrong answers during the consent process are sent to study staff who then contact a potential participant via email or Facebook for further clarification of the consent process. Therefore, no participant will be enrolled that has not answered all consent questions correctly. Participants consenting to participate in the study are asked to verify age by sending proof through email or social media. Verified participants are emailed a baseline assessment. Those completing the baseline assessment are randomized to receive either the Tobacco Status Project (TSP) intervention of referral to the Smokefree.gov website (control).

A stratified random assignment program was developed and participants are assigned from within stratified blocks immediately after completing the baseline assessment. Participants are stratified on stage of change (precontemplation, contemplation, preparation) and smoking pattern (daily vs. non-daily), variables known to be related to outcomes and addressed by the intervention [43]. Participants in the TSP group are assigned to "secret" (private) Facebook groups based on their readiness to quit tobacco.
Groups begin when there are approximately 10 participants enrolled, as that has been deemed the optimal number based on analyses of engagement in our previous work [37] (Thrul J, Klein A, Ramo DE: Smoking cessation intervention on Facebook: Which content generates the best engagement?, submitted). Participants in the control condition are given a referral to the Smokefree.gov program and are encouraged to use it as much as they would like.

Assessments occur online using the survey program Qualtrics at baseline, 3, 6, and 12 months follow-up. Participants receive their choice of gift cards in the amount of $\$ 20$ per assessment, and a $\$ 20$ bonus for completing all three assessments, for a total possible stipend of $\$ 100$ for completing all assessments.

\section{Treatment conditions}

\section{Tobacco Status Project (TSP)}

The TSP is a smoking cessation intervention implemented entirely through "secret" (Facebook's word for entirely private) Facebook groups. All TSP participants are assigned to a Facebook group based on their TTM stage of change (precontemplation: not ready to quit in the next 6 months; contemplation: intending to quit in the next 6 months; or preparation: ready to quit in the next month and demonstrating at least one past year quit attempt).

The group-based intervention has three main features. First, evidence-based strategies have been used to design Facebook posts to be delivered each day for 90 days to intervention groups on Facebook. Posts are based on the US Clinical Practice Guidelines for smoking cessation [2] and the Transtheoretical Model (TTM) of behavior change [44], both recommending treatment be tailored to participants' readiness to quit, and have been determined to be helpful to and likable by young adults (Ramo DE, Chavez K, Delucchi KL, Prochaska JJ: Feasibility and quit rates of the "Tobacco Status Project", submitted). Precontemplation group posts are based on Motivational Interviewing (MI), a directive patientcentered counseling intervention recommended by the Clinical Practice Guidelines [2, 45]. Posts elicit clients' motivation and importance of changing tobacco use, problems associated with use, and using open-ended questions to elicit "change talk" (a client's mention of desire, ability, reason, or commitment to change) through using the 5-R's: relevance, risks, rewards, roadblocks, and repetition, shown to increase likelihood of tobacco quit attempts [46, 47]. Contemplation group posts incorporate MI and the TTM processes of self-liberation (e.g., making a commitment to quit), stimulus control (e.g., removing smoking paraphernalia from the home), and counter conditioning (e.g., engaging in alternative behaviors) are emphasized. Preparation group posts incorporate skills from cognitive behavioral therapy, found effective for long-term smoking cessation [48], as well as 
the TTM processes of self-liberation (e.g., making a commitment to quit), stimulus control (e.g., removing smoking paraphernalia from the home), and counter conditioning (e.g., engaging in alternative behaviors). Posts also encourage setting a quit date and making a detailed quit plan. Facebook posts include a combination of images, videos, text and polls designed to reflect the experience of young adults and all elicit a response from participants. Posts may suggest that participants use their FB or real social networks for support with alcohol or tobacco reduction. However, they are not required to share any information about substance use on social media. Figure 2 provides sample posts for each stage of change.

Second, the intervention incorporates weekly "The Dr. Is In" live sessions with a PhD level smoking cessation counselor (using FB commenting features), during which a counselor provides some limited content for discussion and participants can ask questions and get supplemental support. Content for sessions is tailored to readiness to quit tobacco and is based on Motivational Interviewing (MI) and cognitive behavioral coping skills for smoking cessation.

Third, six manualized 45-min state-of-the-art smoking cessation counseling sessions based on cognitive behavioral therapy (CBT) were adapted for social media delivery for this study. These sessions are designed to be delivered in Facebook events and events are scheduled in each group separately. In contrast to The Dr. Is In sessions that are conversational and question and answer-based, these sessions present more material, and are designed to help participants who are motivated to quit. Thus, only participants in preparation groups are offered these CBT sessions and participants may opt to participate in the sessions at any time during the 90 days of Facebook intervention. In all groups, links to more intensive treatment in a participant's' area are available as needed.

Additionally, we are testing an incentive structure in which the TSP groups are randomized to one of four incentive conditions tied to engagement in the intervention: 1) daily; 2) weekly; 3) monthly; or 4) no incentive. Participants in daily, weekly, or monthly groups can earn money based on comments made to daily Facebook posts at the end of each period. At the end of the 90-day intervention, all participants in paid incentive conditions receive a gift card in the amount they have earned up to $\$ 90$. This is separate from the $\$ 100$ participants could earn for completing all study assessments.

\section{Control group}

Participants in the control condition, receive a referral to the National Cancer Institute's Smokefree.gov website. This website, created by the Tobacco Control Research Branch of the National Cancer Institute, provides information and support to quit smoking for people at all stages of

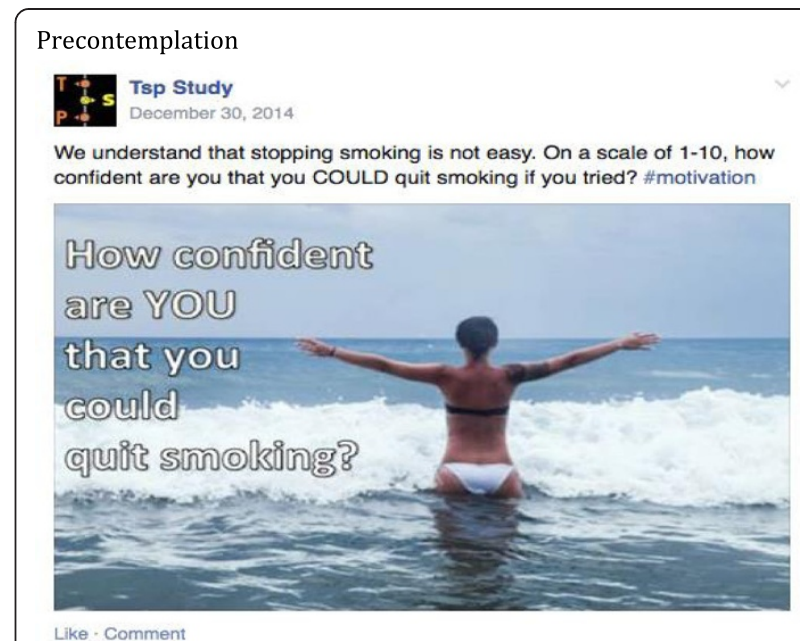

\section{Contemplation

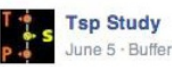

68. One of the best ways to combat a nicotine craving is to keep your hands busy! How do you plan to keep your hands occupied when you get hit with a sudden urge to smoke? \#beingconscious

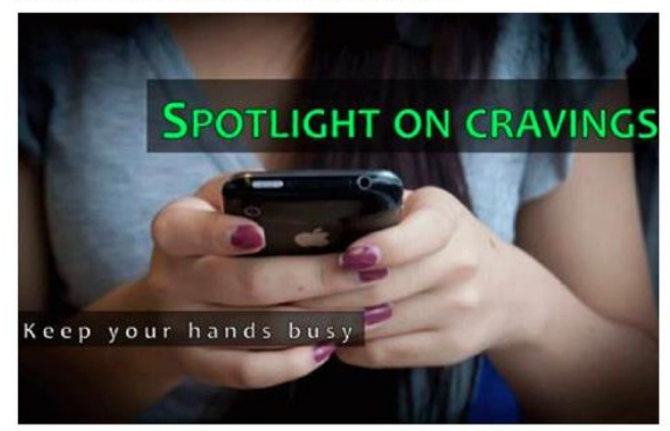

14. Lilke Comment

Preparation

\section{S Tsp Study}

Post what happened when you told your one (or a few) friends about your plan to quit. "support

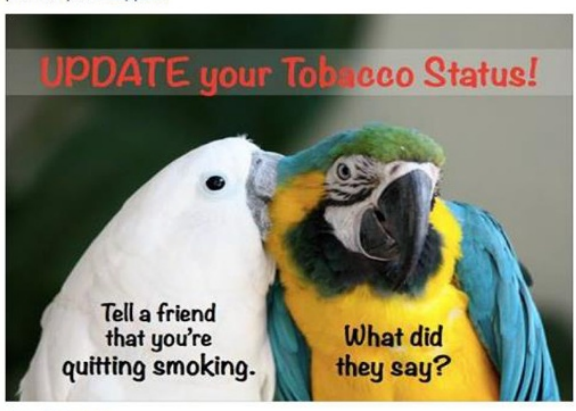

Fig. 2 Tobacco Status Project Sample Posts

change. Smokefree.gov (http://www.smokefree.gov) provides free, accurate, evidence-based information and professional assistance to help support the immediate 
and long-term needs of people trying to quit smoking. Features of the program include a website tailored to readiness to quit smoking, a texting program, Smartphone application, online live chat, and a Facebook page. The treatment provided to control participants meets U.S. Clinical Practice Guidelines for treating nicotine dependence with adults [49].

\section{Measures}

\section{Primary outcome}

The primary outcome biochemically-verified 7-day point prevalence abstinence at 3, 6, and 12 months. Participants reporting "no smoking, not even a puff" in the past 7 days will be coded as abstinent from cigarettes. At each follow-up assessment, participants reporting 7-day abstinence will be mailed a saliva cotinine test kit and asked to record two pictures: one giving a saliva sample, and another of the test result. Participants with a salivary cotinine level $<11 \mathrm{ng} / \mathrm{ml}$, indicating nonsmoking [50], will be considered confirmed nonsmokers. If participants indicate use of an electronic nicotine delivery-system to aid in smoking cessation, saliva cotinine and reported ENDS use will be recorded and reported separately from biochemically verified abstinence.

\section{Secondary outcomes}

The following secondary outcomes will be assessed at each timepoint:

- Reduction of cigarette consumption by $50 \%$ or more $(\mathrm{y} / \mathrm{n})$ between baseline each follow-up will be calculated from the number of cigarettes smoked in the past 7 days at each time point.

- Tobacco quit attempt (y/n). A Follow-up Smoking Questionnaire will assess the presence and number of 24 h quit attempts since the last assessment, used to calculate presence of at least one quit attempt in the assessment time period.

- Readiness to quit tobacco will be assessed using the Stages of Change Questionnaire, [44] categorizing participants into five stage categories at each timepoint (precontemplation, contemplation, preparation, action, and maintenance), and predictive of quit attempts and cessation [51]. Outcome will be measured as proportion in action or maintenance stage sof change at 3,6 , and 12 month assessments.

- Abstinence goal will be assessed with the Thoughts About Abstinence Form, [52], categorizing goal as no goal, intermediary goal (e.g., reduced smoking), or total abstinence. Outcome will be measured as proportion endorsing a goal of abstinence at each timepoint.
- Engagement in TSP will be measured by total number of comments to Facebook study groups during the three-month intervention period.

\section{Power calculation}

Previous trials of stage-based interventions for smoking cessation [53-57] and trials of Internet interventions for smokers motivated to quit $[11,58]$ suggest that quit rates for motivated smokers may be as high as $20 \%$ (treatment) and $9 \%$ (control). However, as this will be the first stagebased intervention for young adult smokers delivered over Facebook, and cessation rates in some Internet trials have been as low as $7 \%$ point prevalence abstinence at 3 month follow-up [59], the conservative estimate of $10 \%$ (treatment) and $5 \%$ (control) 7 -day point prevalence abstinence at 3, 6, and 12 months follow-up was made. Further, attrition in Internet cessation trials has tended to be as high as $50-75 \%$ at 3 and 6 months follow-up [11, 12, 59]. With $50 \%$ attrition at each time point, and at least one covariate (depending on group differences), a sample size of 480 will provide a minimal power level of .80 , and a Type-I error rate of .05 to detect these cessation rates in the most conservative outcome of the study - 7-day point prevalence abstinence.

\section{Data analysis \\ Primary analyses}

Preliminary analyses will describe and summarize all measures and test the correlation between order of study entry and outcome rates of dropout by condition. If differences for any of these variables are noted, they will be statistically controlled as covariates in model testing. Missing data will be minimized through online assessment. When subjects do not complete online assessments, they will be re-contacted through Facebook or email to obtain missing information. For each hypothesis test, two sets of outcome analyses will be conducted - one with all participants who are maintained in the study, and another based only on biochemically verified smoking abstinence rates to allow for direct comparison of findings with the research literature.

The primary outcome of biochemically-verified 7-day abstinence will be compared between participants in the TSP and Control groups at 3-through 12-months follow up using a mixed-effects statistical model $[60,61]$. The independent variables will be intervention versus the control condition, assessment point, plus covariates identified in preliminary analysis. The model will be estimated using maximum likelihood estimation.

\section{Secondary analyses}

Mixed effects logistic and multinomial regression models for longitudinal ordinal response data to model secondary outcomes for tobacco use and across time (3, 6, 12 months): 
1) reduction of cigarettes by $50 \%$ or more $(y / n), 2)$ tobacco quit attempt (y/n; 2 models), 3) action or maintenance stage of change $(\mathrm{y} / \mathrm{n})$; and 4) commitment to abstinence $(\mathrm{y} / \mathrm{n})$. Independent variables in all models will be treatment condition, abstinence status, and covariates identified as relevant to smoking characteristics in the literature.

To evaluate the secondary aim (effect of a monetary incentive on engagement in the TSP condition), we will test for differences in engagement and primary smoking outcome by incentive groups and the relationship between engagement and primary smoking outcome at 3 months. First, due to expected skewed distribution of the engagement variable (comments), the non-parametric Kruskal-Wallace test will examine overall differences in total number of comments made to the Facebook study group by incentive condition, with test for a linear trend in commenting by incentive frequency. Second, Pearson's chi-square test will examine 7-day point prevalence abstinence by incentive condition. Abstinence rates will be examined by incentive group. Two sets of analyses will be conducted: one for biochemically verified abstinence, and one for reported abstinence. Finally, Mann-Whitney $\mathrm{U}$ tests will compare comments for those abstinent at 3 months to those non-abstinent. Again, two sets of analyses will be conducted (one for biochemically-verified abstinence and one for reported abstinence).

\section{Discussion}

To our knowledge, this is the first clinical trial of a smoking cessation intervention for young adults delivered entirely through Facebook. Intervention through social media is innovative and a particularly good option to reach young adult smokers, given widespread use and integration into the lives of users [19]. Yet intervention using this medium can be complicated with respect to recruitment and study design. For example, formative work with the study population indicated that a substantial minority of young adults, particularly those motivated to quit smoking, would be interested in a smoking cessation intervention delivered through Facebook [35]. Yet, a sample of 79 participants recruited for a feasibility trial was primarily white and male, limiting generalizability of the intervention results [36]. In the present trial, efforts are being made through targeted Facebook advertising to recruit a more diverse participant pool.

In this trial, we chose to compare TSP to a control condition in which participants were referred to the Smokefree.gov website. This remains a state-of-the-art digital treatment-as-usual, given scientific basis of its content, and free online access through multiple digital media. Given the overlap in medium and intervention content, significant results will provide strong evidence for the efficacy of TSP. While the content of TSP is similarly based on the Clinical Practice Guidelines, there are differences in the design and specific material presented, and the allFacebook delivery is unique to TSP.

A key concern in using Facebook for intervention is participants' privacy. Social media, by nature, is a public forum for interaction, and there is potential for unintended sharing of information. All TSP intervention components are administered entirely through private groups that are not visible beyond the participants in the groups. Participants are given detailed information about the intervention in the consent process, including notice that all groups are private. However, as in any interaction of social media, data belong to Facebook and their use is dictated by privacy agreements made between each user and Facebook itself, not a research investigator. Participants are made aware during the consent process that their TSP interactions are not completely private and that any concerns should be taken up with the investigators and Facebook itself. All assessments of smoking and other substance use in this study are administered outside of social media (i.e., Qualtrics website housed on a secure UCSF server). The Principal Investigator (first author) has obtained a Federal Certificate of Confidentiality from the NIH to protect all data from subpoena. Investigators have gone to great efforts to ensure that the exchange of information is this trial is used to make positive life changes with the support of an intimate network, and that limitations to privacy and confidentiality are clear to participants.

This trial will help to determine whether and how social media can be harnessed for long-term, biochemically verified smoking cessation in young adults.

\section{Competing interests}

The authors declare that they have no competing interests.

\section{Authors' contributions}

$\mathrm{DR}$ designed the parent study and wrote the protocol in consultation with $\mathrm{KD}, \mathrm{PL}, \mathrm{SH}$, and JP. SH and JP serve as Co-Primary Mentors on the K23 awarded to DR to fund this line of research. KD and PM are Co-Mentors on the award. $J T$ is a postdoctoral fellow on the study, and serves as smoking cessation counselor for the TSP arm of the trial. DR and JT completed the first draft of the manuscript, including all parts, and all authors reviewed and revised subsequent drafts of the manuscript. All authors contributed to and have approved the final manuscript.

\section{Authors' information}

DR is Assistant Professor of Psychiatry at UCSF. JT is a Fellow in the Center for Tobacco Control Research and Education, School of Medicine, UCSF. KD is Professor of Biostatistics in Psychiatry at UCSF. PL is Professor of Medicine at UCSF. SH is Professor of Psychiatry at UCSF. JP is Associate Professor of Medicine with the Stanford Prevention Research Center.

\section{Acknowledgements}

This study was supported by the National Institute on Drug Abuse (NIDA K23 DA032578 and P50 DA09253). Preparation of this manuscript was supported by the National Cancer Institute (CA-113710). None of the funding sources had any further role in study design; in the collection, analysis and interpretation of data; in the writing of the report; or in the decision to submit the paper for publication. 


\section{Author details}

'Department of Psychiatry, University of California, 401 Parnassus Avenue, Box TRC 0984, San Francisco, CA 94143, USA. ${ }^{2}$ Center for Tobacco Control Research and Education, University of California, 530 Parnassus Avenue, San Francisco, CA 94143, USA. ${ }^{3}$ Division of General Internal Medicine, University of California, 1545 Divisadero Street, San Francisco, CA 94143, USA. ${ }^{4}$ Department of Medicine, Stanford Prevention Research Center, Stanford University, 1265 Welch Road, Stanford, CA 94305, USA.

\section{Received: 5 August 2015 Accepted: 2 September 2015}

\section{Published online: 15 September 2015}

\section{References}

1. Jamal A, Agaku IT, O'Connor E, King BA, Kenemer JB. Current cigarette smoking among adults aged-United States, 2005-2013. In: MMWR Morb Mortal Wkly Rep. vol. 63. 2014. p. 1108-12.

2. Fiore MC, Jaén CR, Baker TB, Bailey WC, Benowitz NL, Curry SJ et al. Treating Tobacco Use and Dependence: 2008 Update. Clinical Practice Guideline. Rockville, MD: U.S. Department of Health and Human Services. Public Health Service; 2008

3. Substance Abuse and Mental Health Services Administration: Results from the 2013 National Survey on Drug Use and Health: Table 2.24B - Cigarette Use in Lifetime, Past Year, and Past Month among Persons Aged 18 to 25, by Demographic Characteristics: Percentages, 2012 and 2013. In. Edited by Department of Health and Human Services. Rockville. MD: Substance Abuse Mental Health Services Administration; 2014

4. U. S. Department of Health and Human Services: Preventing tobacco use among youth and young adults: A report of the Surgeon General. In. Atlanta, GA: U. S. Department of Health and Human Services, Centers for Disease Control and Prevention, National Center for Chronic Disease Prevention and Health Promotion, Office on Smoking and Health; 2012.

5. U. S. Department of Health and Human Services: The Health Consequences of Smoking - 50 Years of Progress: A Report of the Surgeon General. In. Atlanta, GA: U. S. Department of Health and Human Services, Centers for Disease Control and Prevention, National Center for Chronic Disease Prevention and Health Promotion, Office on Smoking and Health; 2014.

6. Curry SJ, Sporer AK, Pugach O, Campbell RT, Emery S. Use of tobacco cessation treatments among young adult smokers: 2005 National Health Interview Survey. Am J Public Health. 2007;97(8):1464-9.

7. Bost ML. A descriptive study of barriers to enrollment in a collegiate health assessment program. J Community Health Nurs. 2005;22:15-22.

8. Davies J, McCrae BP, Frank J, Dochnahl A, Pickering T, Harrison B, et al. Identifying male college students' perceived health needs, barriers to seeking help, and recommendations to help men adopt healthier lifestyles. J Am Coll Health. 2000:48(6):259-67.

9. Kramer JJ, Willemsen MC, Conijn B, van Emst AJ, Brunsting S, Riper H. Effectiveness of a web-based self-help smoking cessation intervention: protocol of a randomised controlled trial. BMC Public Health. 2009;9:32.

10. Bock BC, Graham AL, Whiteley JA, Stoddard JL. A review of web-assisted tobacco interventions (WATIs). J Med Internet Res. 2008;10(5):e39.

11. Swartz LH, Noell JW, Schroeder SW, Ary DV. A randomised control study of a fully automated internet based smoking cessation programme. Tob Control. 2006;15(1):7-12.

12. McKay HG, Danaher BG, Seeley JR, Lichtenstein E, Gau JM. Comparing two web-based smoking cessation programs: Randomized controlled trial. J Med Internet Res. 2008;10(5):e40

13. Eysenbach $\mathrm{G}$. Issues in evaluating health Web sites in an Internet-based randomized controlled trial. J Med Internet Res. 2002;4:e17.

14. Feil EG, Noell J, Lichtenstein E, Boles SM, McKay HG. Evaluation of an Internet-based smoking cessation program: Lessons learned from a pilot study. Nicotine Tobacco Res. 2003;5:189-94.

15. Klatt C, Berg CJ, Thomas JL, Ehlinger E, Ahluwalia JS, An LC. The role of peer e-mail support as part of a college smoking-cessation website. Am J Prev Med. 2008;35(6 Suppl):S471-8.

16. An LC, Perry CL, Lein EB, Klatt C, Farley DM, Bliss RL, et al. Strategies for increasing adherence to an online smoking cessation intervention for college students. Nicotine Tob Res. 2006;8 Suppl 1:S7-12.

17. Hu M-C, Davies M, Kandel DB. Epidemiology and correlates of daily smoking and nicotine dependence among young adults. Am J Public Health. 2006;96(2):299-308
18. Lawrence D, Fagan P, Backinger CL, Gibson JT, Hartman A. Cigarette smoking patterns among young adults aged 18-24 years in the United States. Nicotine Tob Res. 2007;9(6):687-97.

19. Duggan M, Ellison NB, Lampe C, Lenhart A, Madden M. Social Media Update 2014. In. Washington, DC: Pew Internet \& American Life Project; 2015.

20. Christakis NA. 2008. BMJ (Clinical Research ed). 2008:336(7659):1468.

21. Cobb NK, Graham AL, Abrams DB. Social network structure of a large online community for smoking cessation. Am J Public Health. 2010;100(7):1282-9.

22. Simoni JM, Nelson KM, Franks JC, Yard SS, Lehavot K. Are peer interventions for HIV efficacious? A systematic review. AIDS Behav. 2011;15(8):1589-95.

23. Gold J, Pedrana AE, Sacks-Davis R, Hellard ME, Chang S, Howard S, et al. A systematic examination of the use of online social networking sites for sexual health promotion. BMC Public Health. 2011;11:583.

24. Bull SS, Levine DK, Black SR, Schmiege SJ, Santelli J. Social mediadelivered sexual health intervention: a cluster randomized controlled trial. Am J Prev Med. 2012;43(5):467-74.

25. Patrick K, Marshall SJ, Davila EP, Kolodziejczyk JK, Fowler JH, Calfas KJ, et al. Design and implementation of a randomized controlled social and mobile weight loss trial for young adults (project SMART). Contemp Clin Trials. 2014;37(1):10-8.

26. Young SD. Social Media Technologies for HIV Prevention Study Retention Among Minority Men Who Have Sex with Men (MSM). AIDS Behav. 2013;18(9):1625-9.

27. Kernot J, Olds T, Lewis LK, Maher C. Effectiveness of a facebook-delivered physical activity intervention for post-partum women: a randomized controlled trial protocol. BMC Public Health. 2013;13:518.

28. Cavallo DN, Tate DF, Ries AV, Brown JD, DeVellis RF, Ammerman AS. A social media-based physical activity intervention: a randomized controlled trial. Am J Prev Med. 2012:43(5):527-32.

29. Napolitano MA, Hayes S, Bennett GG, Ives AK, Foster GD. Using facebook and text messaging to deliver a weight loss program to college students. Obesity. 2013:21(1):25-31.

30. Pedrana A, Hellard M, Gold J, Ata N, Chang S, Howard S, et al. Queer as $F^{* *} \mathrm{k}$ : reaching and engaging gay men in sexual health promotion through social networking sites. J Med Internet Res. 2013;15(2):e25.

31. Williams G, Hamm MP, Shulhan J, Vandermeer B, Hartling L. Social media interventions for diet and exercise behaviours: a systematic review and meta-analysis of randomised controlled trials. BMJ Open. 2014;4(2):e003926.

32. Jones K, Baldwin KA, Lewis PR. The potential influence of a social media intervention on risky sexual behavior and Chlamydia incidence. J Community Health Nurs. 2012;29(2):106-20.

33. Valle CG, Tate DF, Mayer DK, Allicock M, Cai J. Exploring Mediators of Physical Activity in Young Adult Cancer Survivors: Evidence from a Randomized Trial of a Facebook-Based Physical Activity Intervention. J Adolescent Young Adult Oncol. 2015;4(1):26-33.

34. Baskerville NB, Azagba S, Norman C, McKeown K, Brown KS: Effect of a Digital Social Media Campaign on Young Adult Smoking Cessation. Nicotine Tob Res 2015 [Epub ahead of print].

35. Ramo DE, Liu H, Prochaska JJ. A Mixed-Methods Study of Young Adults' Receptivity to Using Facebook for Smoking Cessation: If You Build It, Will They Come? Am J Health Promot. 2014;29(4):e126-35.

36. Ramo DE, Rodriguez TMS, Chavez K, Sommer M, Prochaska JJ. Facebook Recruitment of Young Adult Smokers for a Cessation Trial: Methods, Metrics, and Lessons Learned. Internet Interventions. 2014;1(2):58-64. doi: 10.1016/ j.invent.2014.05.001

37. Ping Q, Yang C Ramo DE. Engagement in a Facebook smoking cessation intervention for young adults: Effects of motivation and monetary incentive. Society for Research on Nicotine and Tobacco; POS4-150. Availabe from: http://www.srnt.org/SRNT_2015_Abstracts_WEB.pdf

38. Substance Abuse and Mental Health Services Administration: Results from the 2013 National Survey on Drug Use and Health: Summary of National Findings. In. Edited by Department of Health and Human Services, vol. NSDUH Series H-48, HHS Publication No. (SMA) 14-4863. Rockville. MD: Substance Abuse Mental Health Services Administration; 2014.

39. Ramo DE, Prochaska JJ. Broad reach and targeted recruitment using Facebook for an online survey of young adult substance use. J Med Internet Res. 2012;14(1):e28.

40. Ramo DE, Hall SM, Prochaska JJ. Reaching young adult smokers through the Internet: Comparison of three recruitment mechanisms. Nicotine Tob Res. 2010;12(7):768-75. 
41. Dunn LB, Kim DS, Fellows IE, Palmer BW. Worth the risk? Relationship of incentives to risk and benefit perceptions and willingness to participate in schizophrenia research. Schizophr Bull. 2009;35(4):730-7.

42. Palmer BW, Cassidy EL, Dunn LB, Spira AP, Sheikh Jl. Effective use of consent forms and interactive questions in the consent process. IRB. 2008;30(2):8-12.

43. Sargent JD, Mott LA, Stevens M. Predictors of smoking cessation in adolescents. Arch Pediatr Adolesc Med. 1998;152(4):388-93.

44. Prochaska JO, DiClemente CC. Stages and processes of self-change for smoking: Toward an integrative model of change. J Consult Clin Psychol. 1983:51(3):390-5.

45. Miller WR, Rollnick S. Motivational Interviewing: Preparing people for change. 2nd ed. New York, NY: Guilford; 2002.

46. Carpenter MJ, Hughes JR, Solomon LJ, Callas PW. Both smoking reduction with nicotine replacement therapy and motivational advice increase future cessation among smokers unmotivated to quit. J Consult Clin Psychol. 2004;72:371-81.

47. Carpernter $M$, Hughes JR, Keely J. Effect of smoking reduction on later cessation: A pilot experimental study. Nicotine Tob Res. 2003;5:155-62.

48. Hall SM, Humfleet GL, Munoz RF, Reus VI, Prochaska JJ, Robbins JA. Using extended cognitive behavioral treatment and medication to treat dependent smokers. Am J Public Health. 2011;101(12):2349-56.

49. U.S. Public Health Service: Clinical Practice Guideline: Treating Tobacco Dependence: 2008 Update. In. Edited by US Department of Health and Human Services; 2008

50. Jarvis MJ, Tunstall-Pedoe H, Feyerabend C, Vesey C, Saloojee Y. Comparison of tests used to distinguish smokers from nonsmokers. Am J Public Health. 1987;77(11):1435-8.

51. DiClemente CC, Prochaska JO, Fairhurst S, Velicer WF, Velasquez MM, Rossi JS. The process of smoking cessation: An analysis of precontemplation, contemplation, and preparation stages of change. J Consult Clin Psychol. 1991;59(2):295-304.

52. Hall SM, Havassy BE, Wasserman DA. Commitment to abstinence and acute stress in relapse to alcohol, opiates, and nicotine. J Consult Clin Psychol. 1990;58(2):175-81.

53. Hollis JF, Polen MR, Whitlock EP, Lichtesteine E, Mullooly JP, Velicer WF, et al. Teen reach: outcomes from a randomized, controlled trial of a tobacco reduction program for teens seen in primary medical care. Pediatrics. 2005;115(4):981-9.

54. Hall SM, Tsoh JY, Prochaska JJ, Eisendrath S, Rossi JS, Redding CA, et al. Treatment for cigarette smoking among depressed mental health outpatients: A randomized clinical trial. Am J Public Health. 2006;96(10):1808-14.

55. Prochaska JO, DiClemente CC, Velicer WF, Rossi JS. Standardized, individualized interactive, and personalized self-help programs for smoking cessation. Health Psychol. 1993;12(5):399-405.

56. Prochaska JO, Velicer WF, Fava JL, Ruggiero L, Laforge RG, Rossie JB, et al. Counselor and stimulus control enhancements of a stage-matched expert system intervention for smokers in a managed care setting. Prev Med. 2001:32(1):23-32

57. Prochaska JO, Velicer WF, Redding C, Rossi JS, Goldstein M, DePue J, et al. Stage-based expert systems to guide a population of primary care patients to quit smoking, eat healthier, prevent skin cancer, and receive regular mammograms. Prev Med. 2005:41(2):406-16.

58. Muñoz RF, Barrera AZ, Delucchi K, Penilla C, Torres LD, Perez-Stable EJ. International Spanish/English Internet smoking cessation trial yields $20 \%$ abstinence rates at 1 year. Nicotine Tob Res. 2009;11(9):1025-34.

59. Cobb NK, Graham AL, Bock BC, Papandonatos G, Abrams DB. Initial evaluation of a real-world Internet smoking cessation system. Nicotine Tob Res. 2005;7(2):207-16.

60. Laird NM, Ware JH. Random-effects models for longitudinal data. Biometrics. 1982;38(4):963-74.

61. Cnaan A, Laird NM, Slasor P. Using the general linear mixed model to analyse unbalanced repeated measures and longitudinal data. Stat Med. 1997;16(20):2349-80

\section{Submit your next manuscript to BioMed Central and take full advantage of:}

- Convenient online submission

- Thorough peer review

- No space constraints or color figure charges

- Immediate publication on acceptance

- Inclusion in PubMed, CAS, Scopus and Google Scholar

- Research which is freely available for redistribution 